\title{
MATLAB GUIDE FOR FORWARD KINEMATIC CALCULATION OF 3 TO 6 DOF SCARA ROBOTS
}

\author{
Suyash Shrivastava ${ }^{1}$ \\ ${ }^{1} P G$ Student, Mechanical Department, Medi Caps Institute of Technology and Management, M.P, India
}

\begin{abstract}
The research work is focused on calculation of position matrices or end effector position of SCARA robotic arm of 3 to 6 Degree of Freedom for various industrial purposes or operations like material handling, assembly, welding, pick and place operations. The calculations of position matrices or end effector position are very complicated and time consuming or laborious, to minimize the time consumption and to enhance learning of forward kinematics of SCARA robot of 3 to 6 DOF a user friendly Graphical User Interface is developed by using MATLAB GUIDE(Graphical User Interface Development Environment ). This MATLAB GUIDE tool is used in calculating position matrices or end effector position of SCARA robots. In this research work 5 and 6 Degree of freedom SCARA robot were developed by referring 3 and 4 degree of freedom robots.
\end{abstract}

Keywords: MATLAB, GUIDE, Forward Kinematics, and SCARA Robots.

\section{INTRODUCTION}

In modern day industries and factories, automation and robotics play a vital role in assembling, material handling, welding, pick and place operations. The accuracy and precision of industrial robot is an important criterion for efficient working. So, it is very important to study kinematics and dynamics of industrial robot for their accurate and precision working.

In this research work SCARA robot is taken for study, by referencing foregoing research work on 3 and 4 Degree of Freedom (DOF) SCARA robots, an idea has developed 5 and 6 DOF SCARA robots. This research work is focused on forward kinematics of 3 to 6 DOF SCARA robots.

MATLAB is a powerful tool used to calculate complex calculations; GUIDE is a part of MATLAB known as Graphical User Interface Development Environment it is used in developing Graphical User Interface.

The research work is based on the Denavit Hartenberg principle, in the Fig.1 Denavit Hartenberg principle is explain and in Table 1 parameters are shown.

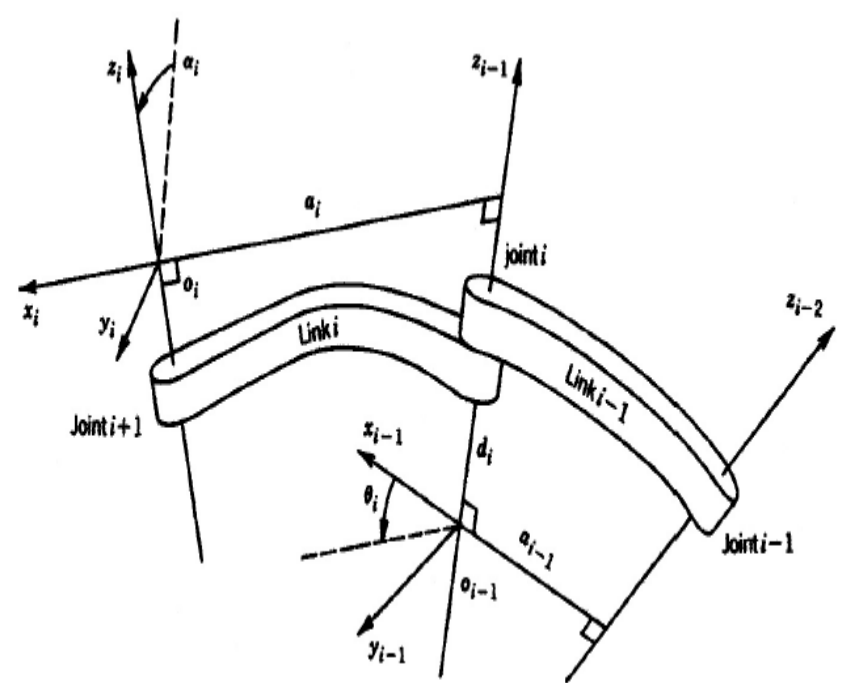

Fig- 1: Denavit Hartenberg Principle

Table- 1: Denavit Hartenberg Parameters

\begin{tabular}{|c|c|c|}
\hline Parameter & Notation & Description \\
\hline $\begin{array}{l}\text { link } \\
\text { length }\end{array}$ & $a_{i}$ & $\begin{array}{l}\text { the offset distance between } \\
Z_{i-1} \text { and } Z_{i} \text { axes along the } x i \\
\text { axis }\end{array}$ \\
\hline $\begin{array}{l}\text { link twist } \\
\text { angle }\end{array}$ & $\alpha_{i}$ & $\begin{array}{l}\text { the angle from the } Z_{i-1} \text { axis to } \\
\text { the } Z_{i} \text { axis about the } x i \text { axis }\end{array}$ \\
\hline $\begin{array}{l}\text { link } \\
\text { offset }\end{array}$ & $d_{i}$ & $\begin{array}{l}\text { the distance from the origin of } \\
\text { frame } i-1 \text { to the } \mathcal{X}_{i} \text { axis along } \\
\text { the } Z_{i-1} \text { axis }\end{array}$ \\
\hline $\begin{array}{l}\text { joint } \\
\text { angle }\end{array}$ & $\theta_{i}$ & $\begin{array}{l}\text { the angle between } \mathcal{X}_{i-1} \text { and } \mathcal{X}_{i} \\
\text { axes about the } Z_{i-1} \text { axis }\end{array}$ \\
\hline
\end{tabular}




\section{OBJECTIVE}

The objective of the research work is to develop two more possible types of SCARA robots for more flexibility and complex machining. The two new possible SCARA robots are 5 and 6 DOF SCARA robot they were developed by referencing 3 and 4 DOF SCARA robots. The major objective is to study the forward kinematics of all the four SCARA robots of 3 to 6 DOF and calculate position matrices. The forward kinematics of SCARA robots were calculated with the help of MATLAB GUIDE tool.

The calculations of forward kinematics of SCARA robot are complex and time consuming, to minimize the problem of calculations and make it easy and less laborious. A user friendly Graphical User Interface is developed by help of MATLAB GUIDE Tool.

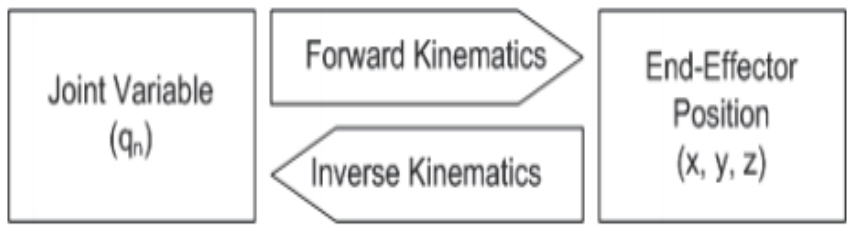

Fig- 2: Forward and Inverse Kinematics

In the Fig 2 one can see relation between forward and inverse kinematics. In forward kinematics position matrices or end effector position are calculated by help of link length and joint angles, and in inverse kinematics joint angles are calculated with the help of link length and end effector position or position matrices. In this research work forward kinematics of SCARA robot is studied and end effector position or position matrices is calculated.

\section{LITERATURE REVIEW}

The Author (Mariappan and Veerabathiram 2016) has done modeling and simulation of multi spindle drilling redundant SCARA robot PRRP (prismatic -revolute -revolute prismatic) which is used to drill multiple holes in printed circuit board and sheet metal, modeling is done using solidworks CAD software and to study dynamic 3D CAD model of robot is converted in Sim-mechanics block diagram by exporting into MATLAB/Sim-mechanics utilizing its motion sensing capability the dynamic parameter velocity and torque of the manipulator are studied and necessary improvement in design of robot is performed. [1]

The Analysts (Chinmayi, et al. 2016) have performed analysis the kinematic of SCARA robot for the application of deburring rectangular path, modeling motion simulation kinematic analysis is done in NX, mathematical analysis is performed in MATLAB, comparison is performed between CAD NX motion simulation kinematics analysis and MATLAB mathematical analysis it is observed there is variation in the time it is because of the change in the direction of movement which can be reduced by providing offset at the point where there is change in the direction. [2]
The Researchers (Venkata Rao, Raju and Subhashini 2014) has performed analysis of SCARA robot for deburring circular profile mathematical model for kinematics and equations are derived from Denavit-Hartenberg notation, kinematic parameter include joint angle, position, and velocities are calculated by modeling and simulation analysis in CAD software and compare with result obtained from MATLAB, it is observed that result match fairly. [3]

The Analysts (Fang and Li 2013) had modeled four degree of freedom SCARA robot simulation and analysis is done by using robotic toolbox in MATLAB. Robot kinematic simulations include both forward kinematics and inverse kinematics, operator has to provide joint angle to the software and robot reach the space coordinate. [4] The Author (Elaikh, et al. 2013) has studied vibration and kinematics characteristic of SCARA robot structure using simulation with the help of MATLAB software. [5]

The Researcher (Shanmugasundar and Sivaramakrishnana 2012) enhanced analytical approach of inverse kinematic SCARA robot by applying mathematical model's linear integration of the parameters by least square method to Adaptive Neuro Fuzzy Network (ANFIS) and the approximation error is back propagated. Matlab is used to simulate the codes and finally ANFIS gives acceptable solution. [6]

The Researcher (Jamali and Shirazi 2012) had modeled, simulated, and optimized virtual prototype of a three degree of freedom SCARA robot by using CAD software solidworks and for multivariable control process and genetic algorithm MATLAB simmechanics is used. [7]

The Author (Jasim 2011) has performed calculation of complex inverse kinematics of four axes SCARA robot by using neuro fuzzy network ANFIS. The reason of using adaptive neuro fuzzy network was, it is easy to operate and errors are in acceptable limit and simulation is quit fast. [8]

The Researcher (Isaksson, et al. 2010) studied kinematic parallel structure of robot to enhance working of SCARA robot, comparison were performed between parallel structure and SCARA robot. [9] The Researcher (Tempea, Neacsa and Livadariu 2009) designed a double SCARA mechanism using CAD software CATIA V5, there are two types of five-bar mechanisms model that have been normalized and reduced, in which the distance between the joints from the frame is zero. In the second case, it offers a new acting solution of active couples, making it easier to build the system. This solution also goes into a simplified forward and inverse kinetametics and simple control system. [10]

\section{SCARA ROBOTS}

There are four SCARA robots they are differentiate on the basis of their varying 3 to 6 DOF or degree of freedom they were discussed below with the help of diagram. 


\subsection{SCARA Robot 3 DOF}

In Fig.3 SCARA robot of three degree of freedom is shown.

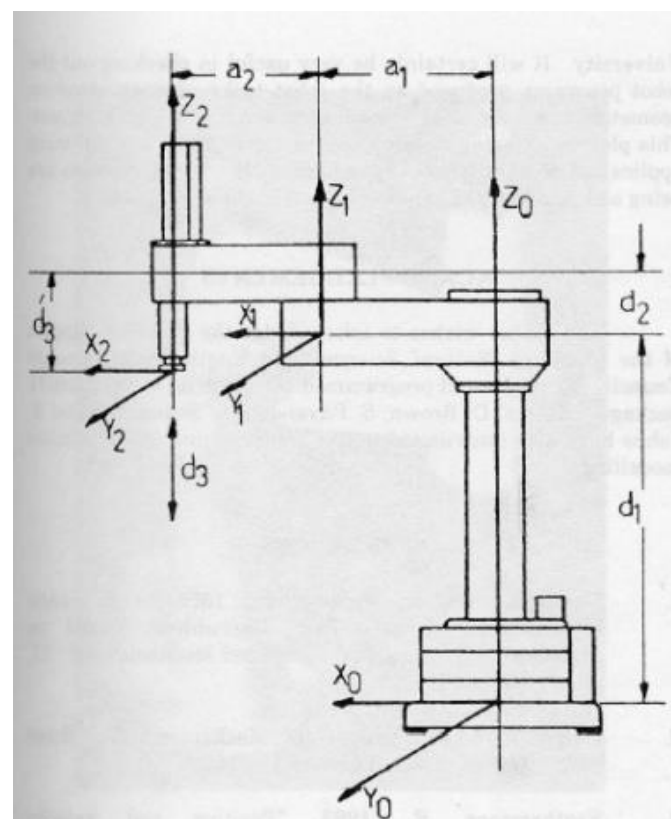

Fig- 3: SCARA Robot 3 DOF

\subsection{SCARA Robot 4 DOF}

In Fig.4 SCARA robot of four degree of freedom is shown.

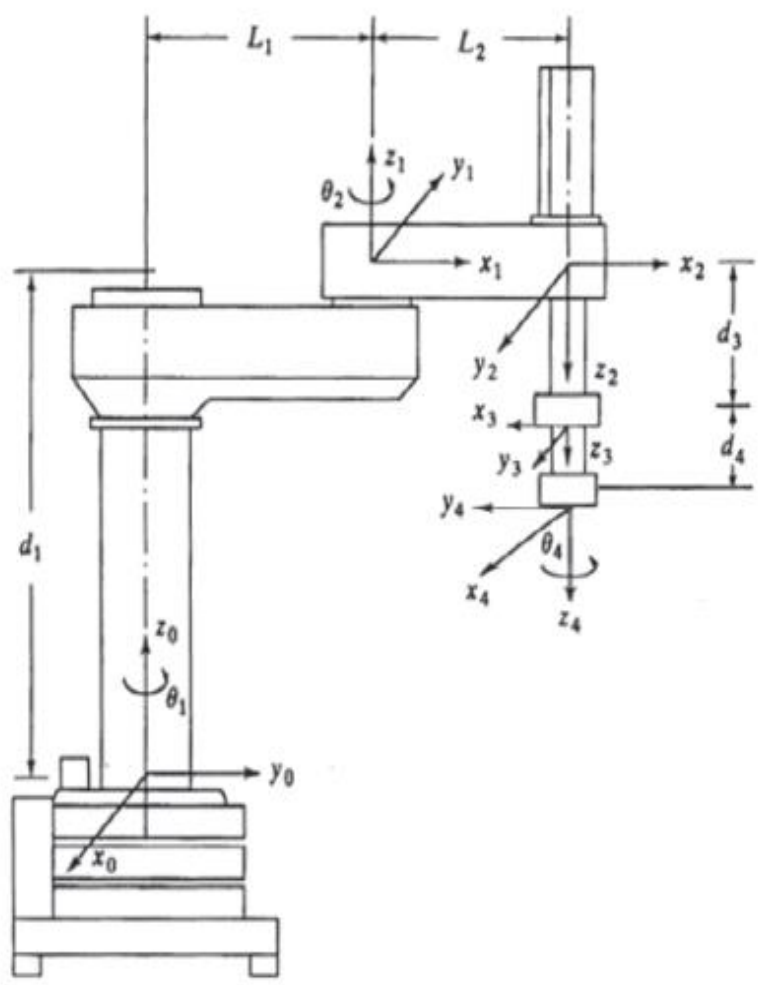

Fig- 4: SCARA Robot 4 DOF

\subsection{SCARA Robot 5 DOF}

In Fig.5 SCARA robot of four degree of freedom is shown.

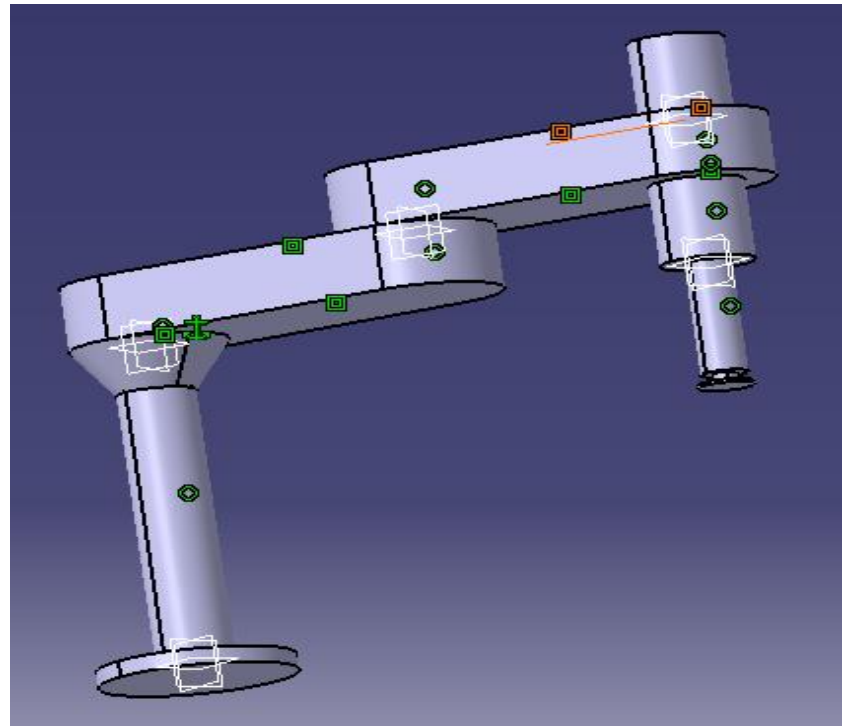

Fig- 5: SCARA Robot 5 DOF

\subsection{SCARA Robot 6 DOF}

In Fig.6 SCARA robot of four degree of freedom is shown.

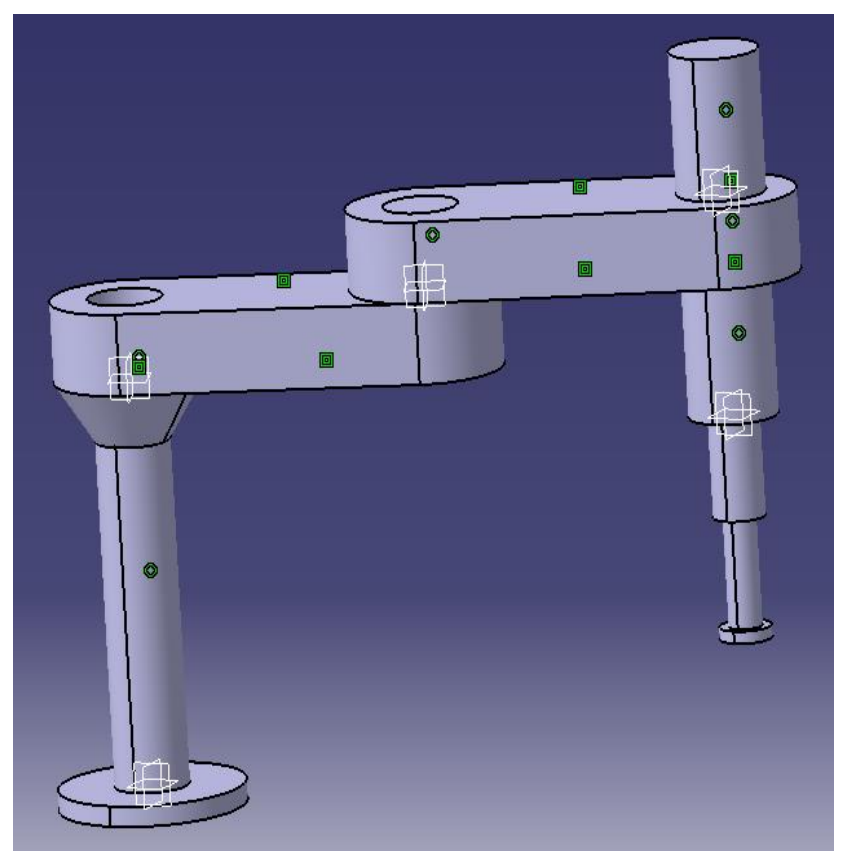

Fig- 6: SCARA Robot 6 DOF

\section{MATHEMATICAL EQUATIONS}

\subsection{SCARA Robot 3 DOF}

Following are the matrices for three degree of freedom SCARA robot

$$
\left\lfloor A_{0}^{1}\right\rfloor=\left[\begin{array}{cccc}
c_{1} & -S_{1} & 0 & a_{1} c_{1} \\
S_{1} & c_{1} & 0 & a_{1} S_{1} \\
0 & 0 & 1 & d_{1} \\
0 & 0 & 0 & 1
\end{array}\right]
$$




$$
\begin{gathered}
{\left[A_{1}^{2}\right]=\left[\begin{array}{cccc}
c_{2} & -s_{2} & 0 & a_{2} c_{2} \\
S_{2} & c_{2} & 0 & a_{2} S_{2} \\
0 & 0 & 1 & d_{2} \\
0 & 0 & 0 & 1
\end{array}\right]} \\
{\left[A_{2}^{3}\right]=\left[\begin{array}{cccc}
c_{3} & -S_{3} & 0 & 0 \\
S_{3} & c_{3} & 0 & 0 \\
0 & 0 & 1 & -d_{3} \\
0 & 0 & 0 & 1
\end{array}\right]} \\
\mathrm{P}_{\mathrm{x}}=\mathrm{a}_{1} \mathrm{C}_{1}+\mathrm{a}_{2} \mathrm{C}_{12}
\end{gathered}
$$

$\mathrm{P}_{\mathrm{Z}}=\mathrm{d}_{1}+\mathrm{d}_{2}-\mathrm{d}_{3}$ (Maraghy 1986) [11]

\subsection{SCARA Robot 4 DOF}

Following are the matrices for four degree of freedom robot

$$
\begin{gathered}
\left\lfloor A_{0}^{1}\right\rfloor=\left[\begin{array}{cccc}
c_{1} & -S_{1} & 0 & a_{1} C_{1} \\
S_{1} & c_{1} & 0 & a_{1} S_{1} \\
0 & 0 & 1 & d_{1} \\
0 & 0 & 0 & 1
\end{array}\right] ; \\
\left\lfloor A_{1}^{2}\right\rfloor=\left[\begin{array}{cccc}
C_{2} & -S_{2} & 0 & a_{2} c_{2} \\
S_{2} & C_{2} & 0 & a_{2} S_{2} \\
0 & 0 & -1 & 0 \\
0 & 0 & 0 & 1
\end{array}\right] ; \\
{\left[A_{2}^{3}\right]=\left[\begin{array}{cccc}
1 & 0 & 0 & 0 \\
0 & 1 & 0 & 0 \\
0 & 0 & 1 & -d_{3} \\
0 & 0 & 0 & 1
\end{array}\right]} \\
\left.A_{3}^{4}\right\rfloor=\left[\begin{array}{cccc}
C_{4} & -S_{4} & 0 & 0 \\
S_{4} & C_{4} & 0 & 0 \\
0 & 0 & 1 & -d_{4} \\
0 & 0 & 0 & 1
\end{array}\right] ; \\
\mathrm{P}_{\mathrm{x}}=\mathrm{a}_{1} \mathrm{C}_{1}+\mathrm{a}_{2} \mathrm{C}_{12}
\end{gathered}
$$$$
\mathrm{P}_{\mathrm{Z}}=\mathrm{d}_{1}-\mathrm{d}_{3}-\mathrm{d}_{4}(\text { Rehiara 2011) [12] }
$$

\subsection{SCARA Robot 5 DOF}

Following are the matrices for five degree of freedom SCARA robot

$$
\begin{aligned}
& \left\lfloor A_{0}^{1}\right\rfloor=\left[\begin{array}{cccc}
c_{1} & -S_{1} & 0 & a_{1} c_{1} \\
S_{1} & c_{1} & 0 & a_{1} S_{1} \\
0 & 0 & 1 & d_{1} \\
0 & 0 & 0 & 1
\end{array}\right] \\
& \left\lfloor A_{1}^{2}\right]=\left[\begin{array}{cccc}
c_{2} & -S_{2} & 0 & a_{2} c_{2} \\
S_{2} & c_{2} & 0 & a_{2} S_{2} \\
0 & 0 & -1 & 0 \\
0 & 0 & 0 & 1
\end{array}\right] \\
& \left\lfloor A_{2}^{3}\right]=\left[\begin{array}{cccc}
1 & 0 & 0 & 0 \\
0 & 1 & 0 & 0 \\
0 & 0 & 1 & -d_{3} \\
0 & 0 & 0 & 1
\end{array}\right] \\
& \left\lfloor A_{3}^{4}\right\rfloor=\left[\begin{array}{cccc}
1 & 0 & 0 & 0 \\
0 & 1 & 0 & 0 \\
0 & 0 & 1 & -d_{4} \\
0 & 0 & 0 & 1
\end{array}\right] \\
& \left\lfloor A_{4}^{5}\right\rfloor=\left[\begin{array}{cccc}
\boldsymbol{C}_{5} & -\boldsymbol{S}_{5} & 0 & 0 \\
\boldsymbol{S}_{5} & \boldsymbol{C}_{5} & 0 & 0 \\
0 & 0 & 1 & -d_{5} \\
0 & 0 & 0 & 1
\end{array}\right] \\
& P_{x}=a_{1} C_{1}+a_{2} C_{12} \\
& P_{y}=a_{1} S_{1}+a_{2} S_{12} \\
& \mathrm{P}_{\mathrm{Z}}=\mathrm{d}_{1}-\mathrm{d}_{3}-\mathrm{d}_{4}-\mathrm{d}_{5}
\end{aligned}
$$

\subsection{SCARA Robot 6 DOF}

Following are the matrices for six degree of freedom SCARA robot

$$
\left[A_{0}^{1}\right]=\left[\begin{array}{cccc}
\boldsymbol{C}_{1} & -S_{1} & 0 & a_{1} \boldsymbol{C}_{1} \\
\boldsymbol{S}_{1} & \boldsymbol{c}_{1} & 0 & a_{1} \boldsymbol{S}_{1} \\
0 & 0 & 1 & d_{1} \\
0 & 0 & 0 & 1
\end{array}\right]
$$




$$
\begin{aligned}
& {\left[A_{1}^{2}\right]=\left[\begin{array}{cccc}
c_{2} & -S_{2} & 0 & a_{2} \boldsymbol{C}_{2} \\
\boldsymbol{S}_{2} & \boldsymbol{C}_{2} & 0 & a_{2} \boldsymbol{S}_{2} \\
0 & 0 & -1 & 0 \\
0 & 0 & 0 & 1
\end{array}\right]} \\
& \left\lfloor A_{2}^{3}\right\rfloor=\left[\begin{array}{cccc}
1 & 0 & 0 & 0 \\
0 & 1 & 0 & 0 \\
0 & 0 & 1 & -d_{3} \\
0 & 0 & 0 & 1
\end{array}\right] ; \\
& \left\lfloor A_{3}^{4}\right\rfloor=\left[\begin{array}{cccc}
1 & 0 & 0 & 0 \\
0 & 1 & 0 & 0 \\
0 & 0 & 1 & -d_{4} \\
0 & 0 & 0 & 1
\end{array}\right] \\
& \left\lfloor A_{4}^{5}\right\rfloor=\left[\begin{array}{cccc}
C_{5} & -S_{5} & 0 & 0 \\
S_{5} & C_{5} & 0 & 0 \\
0 & 0 & 1 & -d_{5} \\
0 & 0 & 0 & 1
\end{array}\right] \\
& \left\lfloor A_{5}^{6}\right]=\left[\begin{array}{cccc}
\boldsymbol{C}_{6} & -\boldsymbol{S}_{6} & 0 & 0 \\
\boldsymbol{S}_{6} & \boldsymbol{C}_{6} & 0 & 0 \\
0 & 0 & 1 & -d_{6} \\
0 & 0 & 0 & 1
\end{array}\right] \\
& P_{x}=a_{1} C_{1}+a_{2} C_{12} \\
& \mathrm{P}_{\mathrm{y}}=\mathrm{a}_{1} \mathrm{~S}_{1}+\mathrm{a}_{2} \mathrm{~S}_{12} \\
& \mathrm{P}_{\mathrm{Z}}=\mathrm{d}_{1}-\mathrm{d}_{3}-\mathrm{d}_{4}-\mathrm{d}_{5}-\mathrm{d}_{6}
\end{aligned}
$$

\section{MATLAB GUIDE WINDOW}

In the Fig.7 the standard view of MATLAB GUIDE WINDOW is shown. In the window one can see all the four parameters of Denavit Hartenberg principle.

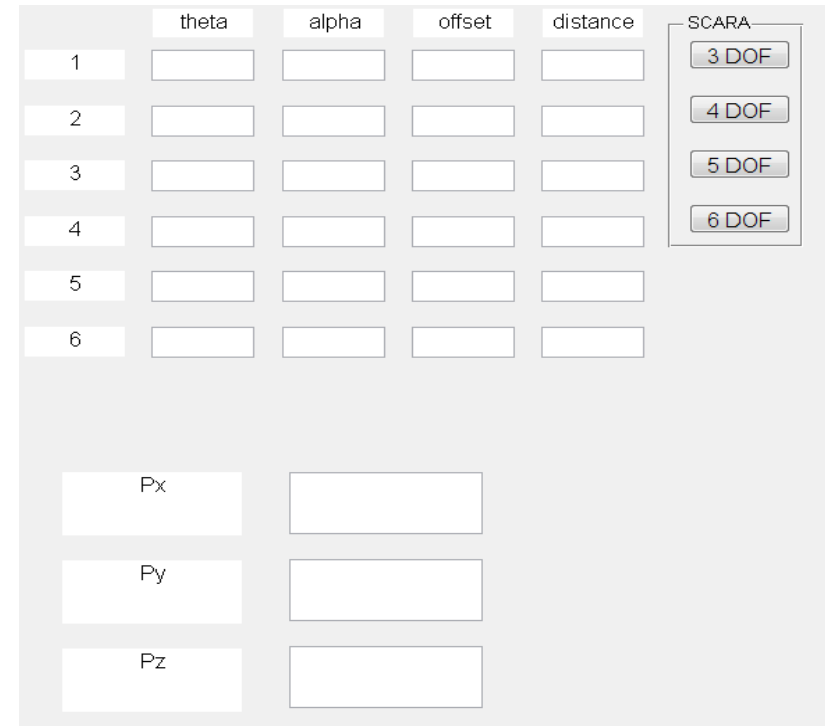

Fig- 7: MATLAB GUIDE WINDOW

There are space provided for individual entry of theta alpha offset and distance. There are separate button provided in the right side of window numbered from 3 to 6 DOF they are responsible for calculation of position matrices or end effector position. The position matrices or end effector position are displayed in the space provided in bottom left side of window.

The windows shown in 6.1 to 6.4 are for calculation of position matrices of SCARA robots.

\subsection{SCARA Robot 3 DOF}

In the SCARA 3 DOF robot we need values of alpha 1 and alpha 2, offset or link length 1 and 2, then we need height or distance of link0, link 2 and link 3 . After that user has to press 3 DOF button and result of position matrices were displayed in space provide bottom left corner of window.

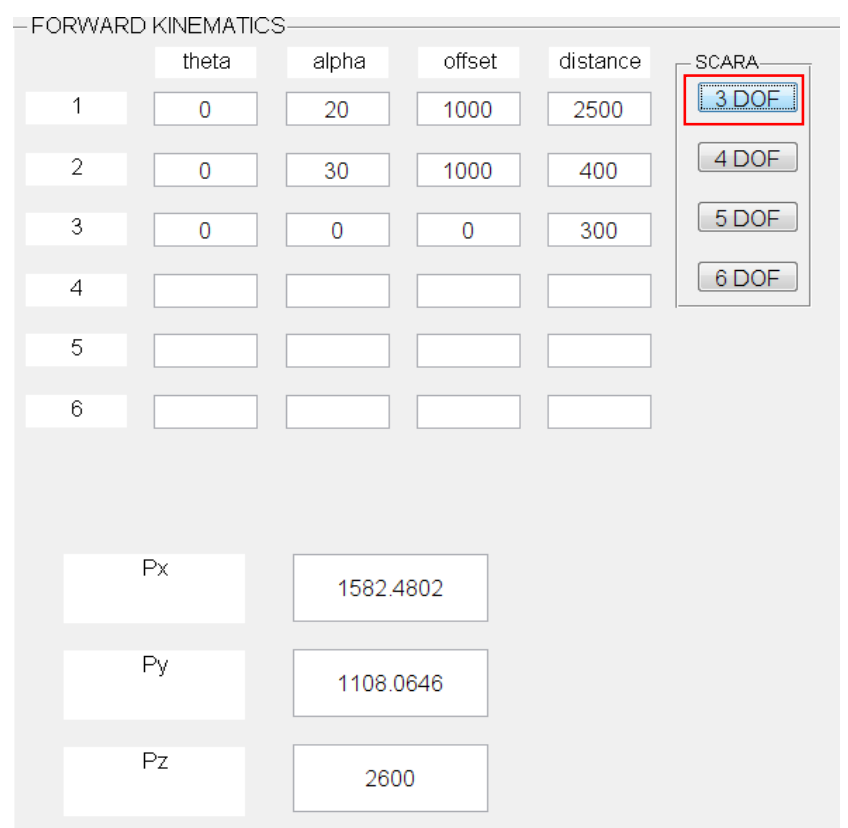

Fig- 8: SCARA Robot 3 DOF 


\subsection{SCARA Robot 4 DOF}

In the SCARA 3 DOF robot we need values of alpha 1 and alpha 2, offset or link length 1 and 2, then we need height or distance of link0, link 2 and link 3 . After that user has to press 3 DOF button and result of position matrices were displayed in space provide bottom left corner of window.

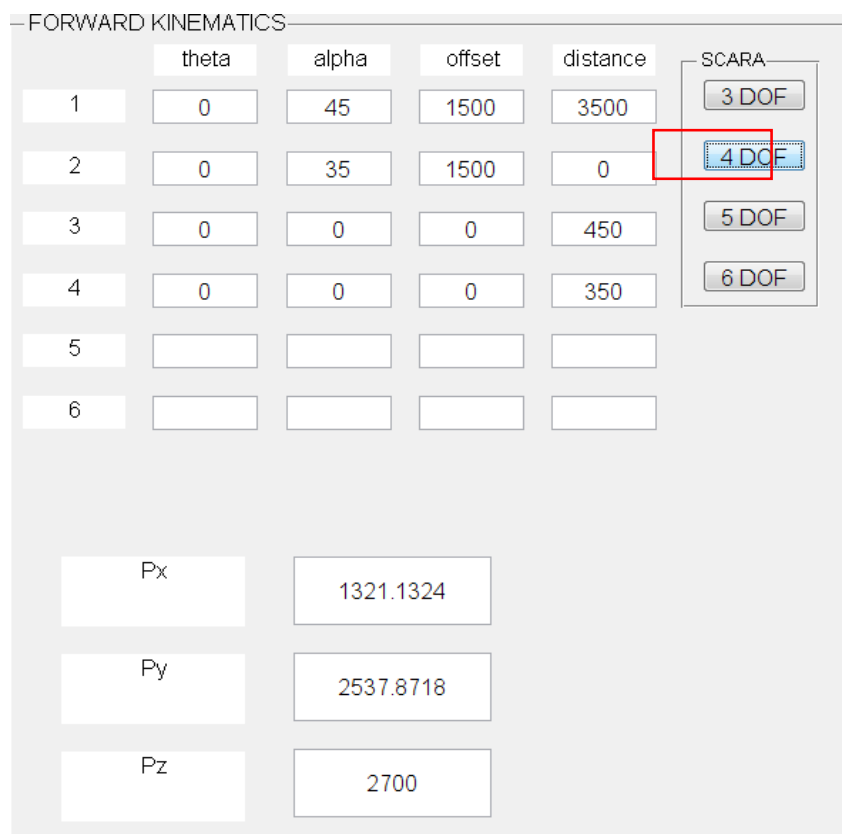

Fig- 9: SCARA Robot 4 DOF

\subsection{SCARA Robot 5 DOF}

In the SCARA 3 DOF robot we need values of alpha 1 and alpha 2, offset or link length 1 and 2, then we need height or distance of link0, link 2 and link 3. After that user has to press 3 DOF button and result of position matrices were displayed in space provide bottom left corner of window.

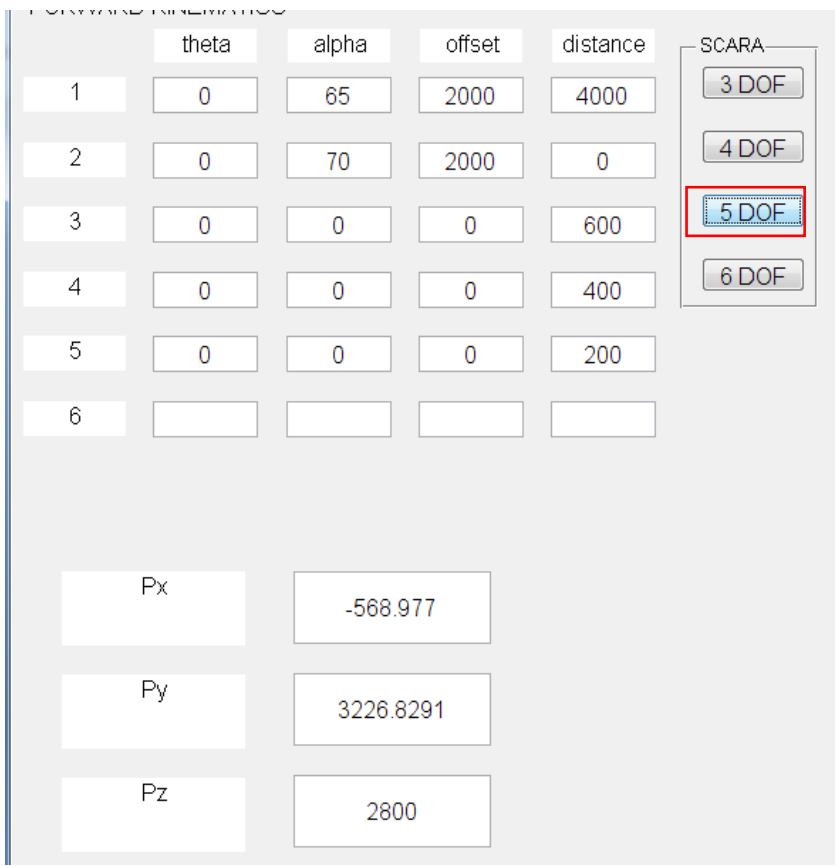

Fig- 10: SCARA Robot 5 DOF

\subsection{SCARA Robot 6 DOF}

In the SCARA 3 DOF robot we need values of alpha 1 and alpha 2, offset or link length 1 and 2 , then we need height or distance of link0, link 2 and link 3. After that user has to press 3 DOF button and result of position matrices were displayed in space provide bottom left corner of window.

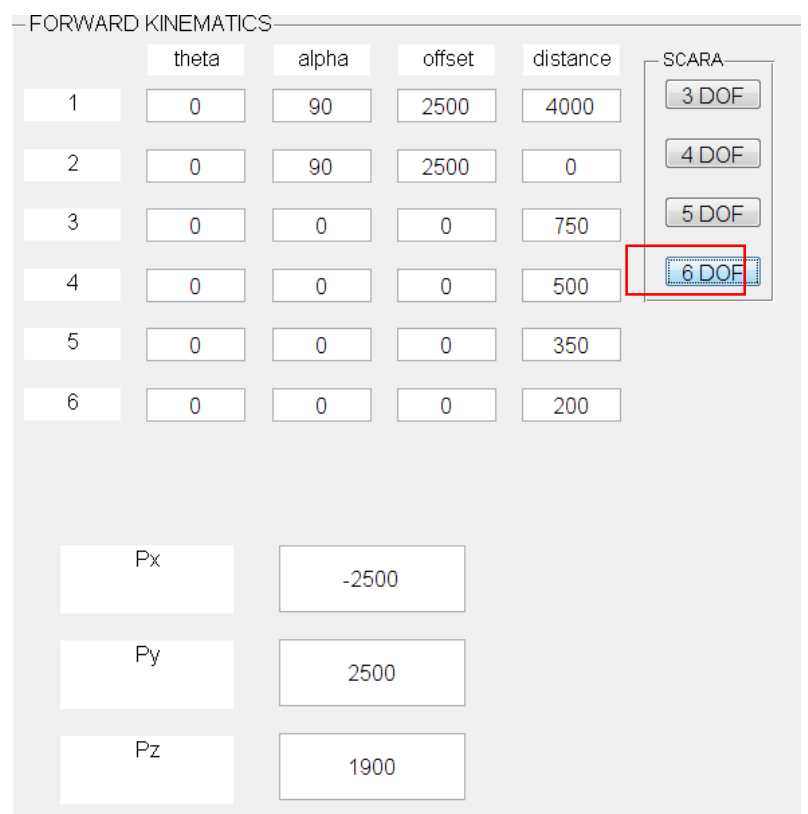

Fig- 11: SCARA Robot 6 DOF

\section{CONCLUSION}

The MATLAB GUIDE software is working perfectly and providing results of position matrices or end effector position for the entire four SCARA robots from 3 to 6 dof robot configurations.

The objective of research work is to develop MATLAB GUIDE a user friendly tool to solve complex and time consuming calculation of position matrices is successfully achieved. The software is working and providing data of position matrices for all the four SCARA robots.

\section{REFERENCES}

[1] Mariappan, Saravana Mohan, and Anbumalar Veerabathiram. "Modelling and Simulation of Multi Spindle Drilling Redundant SCARA Robot using Solidworks and MATLAB/ SimMechanics." Facult of Engineering, University of Antioquia (ResearchGate), December 2016: 63-72.

[2] Chinmayi, k, PVS Subhashini, G Venkata Rao, and NVS Raju. "Kinematic Aanlysis of a SCARA robot for Deburring of Rectangular Paths." International Journal of Scientific \& Engineering Research 7, no. 6 (June 2016): 57-62.

[3] Venkata Rao, G, NVS Raju, and PVS Subhashini. "Modelling Simulation and Analysis of a SCARA Robot for Deburring of Circular Components." APRN Journal of Engineering and Applied Sciences 9, no. 4 (April 2014): 398-404. 
[4] Fang, Jian, and Wei Li. "Four Degree of Freedom SCARA Robot Kinematics Modeling and Simulation Analysis." International Journal of Computer Consumer and Control 2, no. 4 (2013): 20-27.

[5] Elaikh, Talib EH, Haider J Abed, Kadhim M Abed, Salah M Swadi, and Kadhim Karim M. "Vibration and Kinematic Analysis of SCARA Robot Structure." Diyal Joural of Engineering Sciences 6, no. 3 (September 2013): 127-143.

[6] Shanmugasundar, G, and R Sivaramakrishnana. "Software Development for an Inverse Kinematics of Seven degree of Freedom Newly Designed Articulated Inspection Robot." International Journal of Computer Application 58, no. 18 (November 2012): 29-36.

[7] Jamali, Pouya, and Kourosh H Shirazi. "Robot Manipulators Modeling Simulation and Optimal Multi Variable Control." Trans Tech Publications , 2012: 1-5.

[8] Jasim, Wesam Mohammed. "Solution of inverse kinematics for Scara Manipulator using Adaptive Neuro Fuzzy Network." International Journal on Soft Computing 2, no. 4 (November 2011): 59-66.

[9] Isaksson, Mats, Torgny Torgardh, Ivan Lundberg, and Saeid Nahavandi. "Improving the Kinematic Performance of the SCARA Tau PKM." IEEE International Conference on Robotics and Automation, May 2010: 4683-4690.

[10] Tempea, Iosif, Marin Neacsa, and Adriana Livadariu. "A New Acting Solution of a Double SCARA Robot." The 3rd International Conference on "Computational Mechanics and Virtual Engineering" , October 2009: 765-770

[11] Maraghy, H. A. (1986, May 26-30). Kinematic and Geometric Modelling and Animation od Robots. Canadian Conference Information Processing Society , 15-19.

[12] Rehiara, A. B. (2011). Kinematics of Adept Three Robot Arm. Papua Indonesia: Intech.

\section{BIOGRAPHIE}

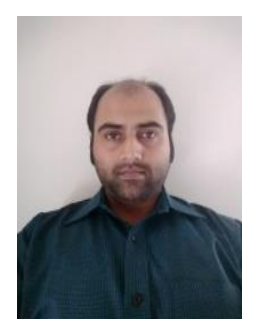

Raipur.
Suyash Shrivastava is Master of Engineering student of Medi-Caps Institute of Technology and Management, Indore specialization in Computer Integrated Manufacturing. He had done his Bachelor of Engineering in Mechanical Department from Central College of Engineering and Management, 